\title{
Photoluminescence and electrical characteristics of the two-dimensional electron gas in Si delta-doped GaN layers
}

\author{
C. W. Teng \\ Department of Electrical and Computer Engineering, North Carolina State University, Raleigh, \\ North Carolina 27695-7911 \\ M. O. Aboelfotoh and R. F. Davis \\ Department of Materials Science and Engineering, North Carolina State University, Raleigh, \\ North Carolina 27695-7907 \\ J. F. Muth ${ }^{\text {a) }}$ and R. M. Kolbas \\ Department of Electrical and Computer Engineering, North Carolina State University, Raleigh, \\ North Carolina 27695-7911
}

(Received 29 August 2000; accepted for publication 15 January 2001)

\begin{abstract}
We have studied the electrical and photoluminescence (PL) properties of a Si delta-doped GaN layer grown by metalorganic chemical vapor deposition. The Hall mobility and electron sheet concentration are $726 \mathrm{~cm}^{2} / \mathrm{V} \mathrm{s}$ and $1.9 \times 10^{12} \mathrm{~cm}^{-2}$, respectively, at $2 \mathrm{~K}$. A PL peak located at 78 $\mathrm{meV}$ below the band gap of $\mathrm{GaN}$ is observed at $77 \mathrm{~K}$. This PL peak is attributed to the radiative recombination between electrons in the two-dimensional quantum states and photoexcited holes in $\mathrm{GaN}$, which is consistent with simulation results using a one-dimensional Poisson and Schrödinger equation solver. The peak disappears at temperatures higher than $77 \mathrm{~K}$ and is not observed in uniformly doped GaN layers. (C) 2001 American Institute of Physics. [DOI: 10.1063/1.1353836]
\end{abstract}

Delta doping of GaAs and other compound semiconductor structures has been intensively studied in the past decade. Confining the dopants to a single or few monolayers of the semiconductor lattice allows one to explore the fundamental aspects of device physics and to fabricate device structures such as delta-doped metal-semiconductor field-effect transistors and $n-i-p-i$ superlattices. ${ }^{1}$ Recent development of high-power and high-speed electronic devices based upon $\mathrm{Al}_{x} \mathrm{Ga}_{1-x} \mathrm{~N} / \mathrm{GaN}$ heterostructures is very promising because of their large band gap energy, high electron peak velocity, and high breakdown electric field., ${ }^{2,3}$ Applying the delta doping technique in III-nitride based high-speed transistor fabrication is especially interesting. Kim et al. recently reported a sharp carrier concentration profile with a peak concentration $9.8 \times 10^{18} \mathrm{~cm}^{-3}$ by measuring the capacitance-voltage characteristics on Si delta-doped GaN layers grown by metalorganic chemical vapor deposition. ${ }^{4}$ So far, however, there has been no report on the electrical or optical properties of deltadoped $\mathrm{GaN}$ layers.

Low-temperature photoluminescence (PL) related to the radiative recombination between the two-dimensional electron gas (2DEG) and photoexcited holes have been useful tools in understanding the quantum confined states of 2DEG at $\mathrm{Al}_{x} \mathrm{Ga}_{1-x} \mathrm{As} / \mathrm{GaAs}$ heterointerfaces ${ }^{5,6}$ and in delta-doped GaAs layers. ${ }^{7}$ Recently, PL studies of the 2DEG have been extended to $\mathrm{Al}_{x} \mathrm{Ga}_{1-x} \mathrm{~N} / \mathrm{GaN}$ heterostructures. For example, Bergmann et al. reported that a broad weak PL peak about $50 \mathrm{meV}$ below the free exciton emission of GaN and interpreted it as arising from the recombination of electrons within the 2DEG at the $\mathrm{Al}_{x} \mathrm{Ga}_{1-{ }_{x}} \mathrm{~N} / \mathrm{GaN}$ heterointerface with photoexcited holes in GaN. ${ }^{8}$ Kwon et al. also identified

${ }^{a)}$ Electronic mail: muth@unity.ncsu.edu a $4 \mathrm{~K}$ PL peak due to the radiative recombination between the 2DEG and holes bound to residual acceptors. ${ }^{9}$ Shen et al., however, found that the intensity of the 2DEG peak could be enhanced significantly by incorporating a thin $\mathrm{Al}_{0.12} \mathrm{Ga}_{0.88} \mathrm{~N}$ layer in the $\mathrm{GaN}$ layer near the heterointerface to suppress the diffusion of photoexcited holes. ${ }^{10}$ In this letter, we report results from a PL study of delta-doped GaN layers. A PL peak located at $78 \mathrm{meV}$ below the band gap of $\mathrm{GaN}$ is observed at $77 \mathrm{~K}$ and is attributed to the recombination between 2DEG and photoexcited holes in GaN.

The Si delta-doped GaN layers were grown by metalorganic chemical vapor deposition using triethylgallium in combination with ammonia, silane, and a $\mathrm{H}_{2}$ diluent. A 100nm-thick AlN buffer layer was first grown on an on-axis $6 \mathrm{H}-\mathrm{SiC}(0001)$ substrate at $1100{ }^{\circ} \mathrm{C}$. This was followed by the growth of an $\sim 1 \mu \mathrm{m}$ unintentionally doped $\mathrm{GaN}$ layer, a 7.5-nm-thick Si doped GaN layer, and then a $0.1 \mu \mathrm{m} \mathrm{GaN}$ capping layer at $1000{ }^{\circ} \mathrm{C}$. The $\mathrm{Si}$ dopant concentration was estimated to be $1 \times 10^{19} \mathrm{~cm}^{-3}$. It has been shown that $\mathrm{Si}$ can change the growth mode of GaN. ${ }^{11}$ However, cross-sectional transmission electron microscopy measurements revealed no change in the growth mode and a relatively low dislocation density in the sample, which is consistent with the PL data shown later. The mobility of the Si delta-doped GaN layers was investigated using Hall measurements in a Van der Pauw configuration at various temperatures and the results are shown in Fig. 1. The ohmic contacts to $\mathrm{GaN}$ were made of $\mathrm{Au}$ and $\mathrm{Al}$ alloyed at $480{ }^{\circ} \mathrm{C}$. The Hall mobility and electron sheet concentration are $726 \mathrm{~cm}^{2} / \mathrm{V} \mathrm{s}$ and 1.9 $\times 10^{12} \mathrm{~cm}^{-2}$, respectively, at $2 \mathrm{~K}$. The Hall mobility decreases with increasing temperature, reaching a value of only $15 \mathrm{~cm}^{2} / \mathrm{V} \mathrm{s}$ at room temperature, which is much lower than the values reported by Götz et al. ${ }^{12}$ on uniformly doped GaN layer with $\mathrm{Si}$ concentration of $\sim 2 \times 10^{19} \mathrm{~cm}^{-3}$ grown by 


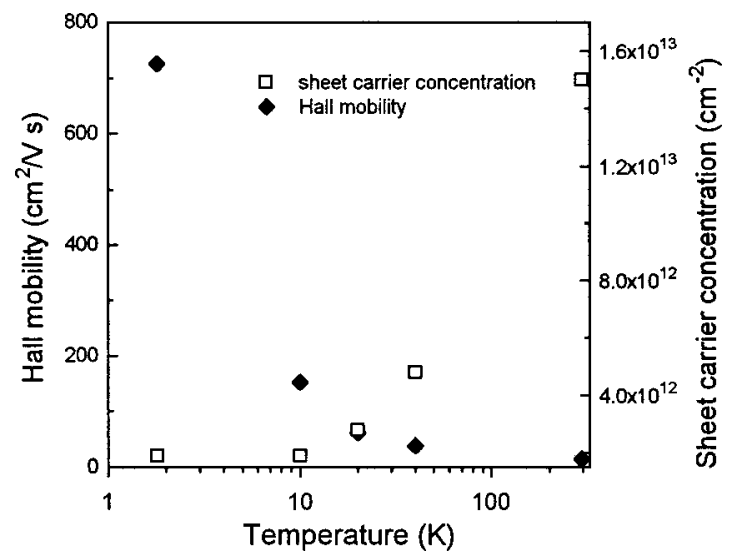

FIG. 1. The Hall mobility and sheet carrier concentration of a Si deltadoped $\mathrm{GaN}$ layer as a function of temperature.

metalorganic vapor phase epitaxy. The low roomtemperature mobility may be attributed at least in part to scattering by ionized impurities or charged defects created during the growth. Nevertheless, the behavior observed here is very similar to that found in $\mathrm{Al}_{x} \mathrm{Ga}_{1-x} \mathrm{~N} / \mathrm{GaN}$ heterostructures. However, it is markedly different from the results reported in Ref. 12 for uniformly doped samples, which show that the mobility reaches a peak value of $135 \mathrm{~cm}^{2} / \mathrm{V} \mathrm{s}$ at 300 $\mathrm{K}$ and then decreases as the temperature is lowered.

The PL spectra were excited at $77 \mathrm{~K}$ with a continuouswave argon ion laser (270-305 nm) and collected using a $0.64 \mathrm{~m}$ spectrometer equipped with an S-20 photocathode. In Fig. 2(a) we show the PL spectrum from a $\mathrm{Si}$ delta-doped $\mathrm{GaN}$ layer. The PL spectrum from a uniformly doped $\mathrm{GaN}$ layer with $\mathrm{Si}$ concentration of $\sim 5 \times 10^{17} \mathrm{~cm}^{-3}(\sim 1 \mu \mathrm{m}$ thick) is also shown in Fig. 2(b) for comparison. The dominant band edge emission $(\sim 360 \mathrm{~nm})$ and a set of deep level emission peaks at 380,391, and $403 \mathrm{~nm}$ are observed in both samples. The full width at half maximum of the band edge emission in the delta-doped sample is approximately 12 $\mathrm{meV}$, indicating very high crystal quality of the layer. In both samples, the peak at $380 \mathrm{~nm}$ is identified as due to donor-shallow-acceptor pair (DAP) recombination. ${ }^{13,14}$ Two LO-phonon replicas (391 and $403 \mathrm{~nm}$ ) at intervals of 92 and $185 \mathrm{meV}$ were observed at $77 \mathrm{~K}$ due to the electron-phonon coupling of the DAP transitions. A relatively weak emission peak at $366.8 \mathrm{~nm}(3.379 \mathrm{eV}), 78 \mathrm{meV}$ below the band edge, is only observed in the delta-doped GaN sample. This peak, however, was not observed when the temperature was raised to higher temperature.

To understand the origin of this peak, the structure was simulated using a one-dimensional Poisson and Schrödinger equation solver. ${ }^{15}$ In the modeling we assumed a $7.5-\mathrm{nm}$ thick delta-doped region with $\mathrm{Si}$ concentration of $1 \times 10^{19}$ $\mathrm{cm}^{-3}$ sandwiched between undoped $\mathrm{GaN}$ layers with residual donor concentration of $1 \times 10^{15} \mathrm{~cm}^{-3}$. The electron and hole effective masses used in the simulations are 0.20 and $1.80 \mathrm{~m}_{0}$, respectively. The simulated band diagram of the delta-doped sample is shown in the inset of Fig. 2(a). The Fermi level $\left(E_{F}\right)$ of the system at equilibrium is $25 \mathrm{meV}$ below the unperturbed $\mathrm{GaN}$ conduction band edge, and the ground quantum confined state energy of the 2DEG is $\sim 53$ $\mathrm{meV}$ below the Fermi level. The transition energy of the radiative recombination between the $2 \mathrm{DEG}$ and free holes at Downloaded 16 Apr 2008 to 152.1.24.180. Redistribution subject

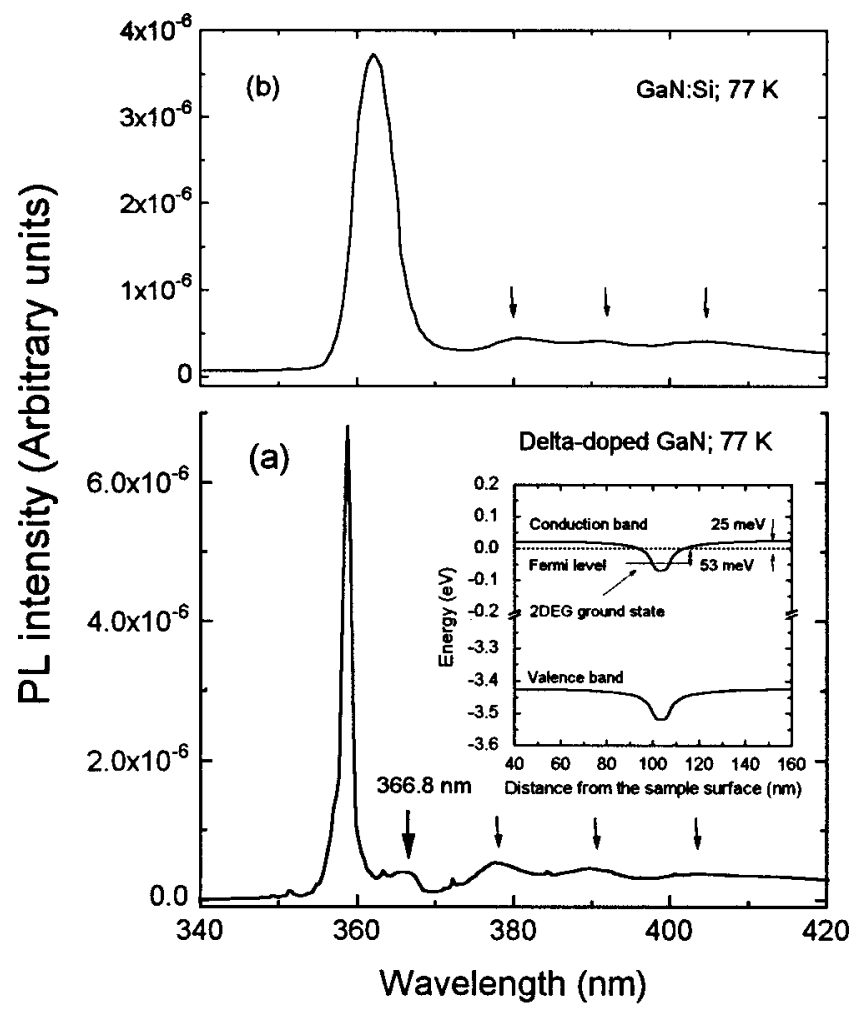

FIG. 2. (a) The PL spectrum of a Si delta-doped GaN layer measured at 77 $\mathrm{K}$. The arrow at $366.8 \mathrm{~nm}$ designates the radiative recombination between electrons in the 2DEG and photoexcited holes. The other three arrows designate the donor-acceptor-pair recombination and its LO-phonon replica. The simulated band diagram of the delta-doped sample by using a onedimensional Poisson and Schrödinger equation solver is shown in the inset. (b) The $77 \mathrm{~K}$ PL spectrum of a uniformly Si-doped GaN sample.

the valence band edge is therefore $78 \mathrm{meV}$ below the band gap of GaN, which is consistent with our PL results. The uncertainties in the transition energies as a result of $\pm 10 \%$ fluctuation of dopant concentration or delta-doped layer thickness are only \pm 4 and $\pm 2 \mathrm{meV}$, respectively, which are within the experimental error range. The effective electric field in a delta-doped structure can be calculated by considering a V-shaped quantum well in the region. The eigenstate energies of a $\mathrm{V}$-shaped quantum well can be solved analytically by using Wentzel-Kramers-Brillouin approximation and are given by ${ }^{1}$

$$
E_{n}=\left[\frac{3}{4}\left(n+\frac{1}{2}\right)\right]^{2 / 3}\left(\frac{e^{2} \hbar^{2} F^{2}}{2 m_{e}^{*}}\right)^{1 / 3} n=0,1,2 \ldots,
$$

where $F$ is the effective electric field, and $E_{n}$ is the $n$th eigenstate energy of 2DEG relative to the bottom of the well. From the simulation the ground state energy is $\sim 16 \mathrm{meV}$ above the bottom of the well, and the effective electric field is calculated to be $3.1 \times 10^{4} \mathrm{~V} / \mathrm{cm}$, which is close to the field value in $\mathrm{Al}_{x} \mathrm{Ga}_{1-x} \mathrm{~N} / \mathrm{GaN}$ heterostructures reported in Ref. 9.

It is interesting to verify the origin of the $366 \mathrm{~nm}$ emission peak from the delta-doped GaN sample by comparing the low temperature PL results with those from $\mathrm{Al}_{x} \mathrm{Ga}_{1-x} \mathrm{~N} / \mathrm{GaN}$ heterostructures. ${ }^{9,10}$ We therefore performed PL measurements on an $\mathrm{Al}_{0.12} \mathrm{Ga}_{0.88} \mathrm{~N} / \mathrm{GaN}$ single heterostructure sample at $77 \mathrm{~K}$. The sample is grown on a $6 \mathrm{H}-\mathrm{SiC}$ substrate. From the bottom to the top, it consists of a $100 \mathrm{~nm}$ AlN buffer layer, a $1.2-\mu \mathrm{m}$-thick undoped GaN layer, a 10 -nm-thick $\mathrm{Al}_{0.12} \mathrm{Ga}_{0.8} \mathrm{~N}$ spacer, a 170-nm-thick
AlP license or copyright; see http://apl.aip.org/apl/copyright.jsp 


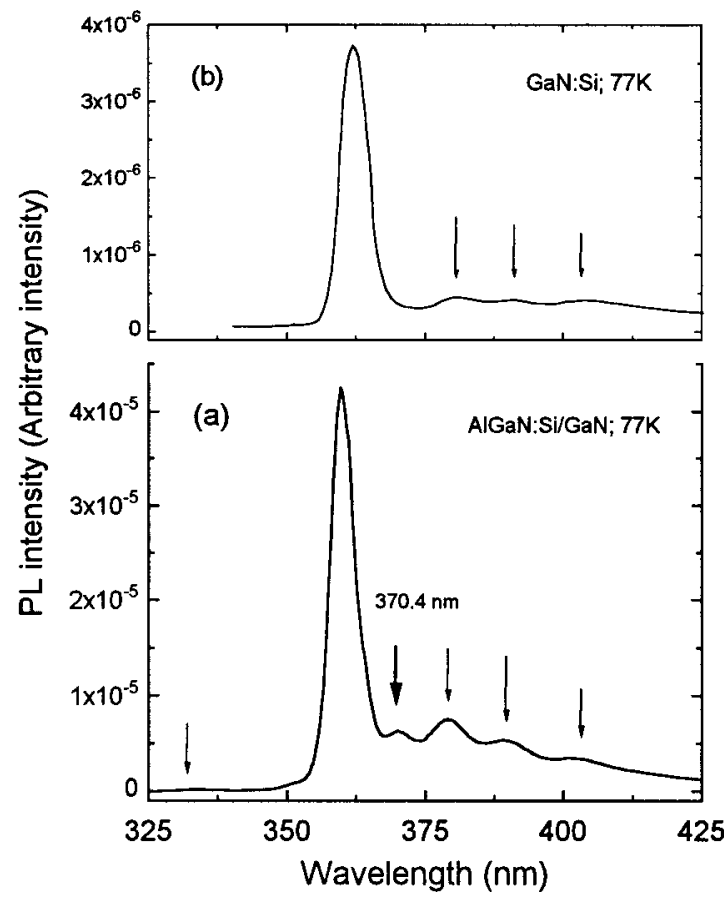

FIG. 3. (a) The PL spectrum of a doped $\mathrm{Al}_{0.12} \mathrm{Ga}_{0.88} \mathrm{~N} / \mathrm{GaN}$ heterostructure at $77 \mathrm{~K}$. (b) The PL spectrum of a uniformly Si-doped GaN sample at $77 \mathrm{~K}$.

$n-\mathrm{Al}_{0.12} \mathrm{Ga}_{0.88} \mathrm{~N}: \mathrm{Si}\left(1 \times 10^{19} / \mathrm{cm}^{3}\right)$, and then capped with a $70-$ nm-thick GaN:Si layer. The fact that the 2DEG were formed at the heterointerface was verified by Hall mobility measurements. The PL spectrum for this heterostructure sample is shown in Fig. 3(a). Similarly, the dominant band edge emission $(361.2 \mathrm{~nm})$ and a set of deep level emission peaks are observed in the uniformly Si-doped GaN [Fig. 3(b)] and the $\mathrm{Al}_{x} \mathrm{Ga}_{1-x} \mathrm{~N} / \mathrm{GaN}$ heterostructure. A weak peak at $\sim 330 \mathrm{~nm}$ is observed and assigned to the emission from the $\mathrm{Al}_{0.12} \mathrm{Ga}_{0.88} \mathrm{~N}$ layer. The $370.4 \mathrm{~nm}$ peak is only observed in the heterostructure sample and is similar to the PL peak reported by Kwon et al. (Ref. 9) on a modulation-doped $\mathrm{Al}_{0.37} \mathrm{Ga}_{0.63} \mathrm{~N} / \mathrm{GaN}$ single heterostructure. As in Ref. 9 we assume the transition results from the radiative recombination of electrons in the 2DEG with holes weakly bound to residual acceptors in GaN. This suggests that lowtemperature PL is a very sensitive tool to explore the quantum confined states of 2DEG in delta-doped GaN layers and modulation-doped $\mathrm{Al}_{x} \mathrm{Ga}_{1-x} \mathrm{~N} / \mathrm{GaN}$ heterostructures.

In summary, the electrical and PL properties of a $\mathrm{Si}$ delta-doped GaN layer grown by metalorganic chemical vapor deposition were investigated. The Hall mobility and electron sheet concentration are $726 \mathrm{~cm}^{2} / \mathrm{V} \mathrm{s}$ and $1.9 \times 10^{12}$ $\mathrm{cm}^{-2}$, respectively, at $2 \mathrm{~K}$. A PL peak located at $78 \mathrm{meV}$ below the band gap of $\mathrm{GaN}$ is observed at $77 \mathrm{~K}$, and is attributed to the radiative recombination between electrons in the two-dimensional quantum states and photoexcited holes. A comparative study was performed on uniformly Si-doped $\mathrm{GaN}$ and $\mathrm{Al}_{x} \mathrm{Ga}_{1-x} \mathrm{~N} / \mathrm{GaN}$ heterostructures to verify that the luminescence is indeed related to the two-dimensional electron states. The experimental results are consistent with those from simulations using a one-dimensional Poisson and Schrödinger equation solver.

Two of the authors (M.O.A. and R.F.D.) express their appreciation to the Office of Naval Research for their support of this research under Grant Nos. N00014-92-J-1500 and N00014-92-J-1080 and to Cree Research, Inc. for $6 \mathrm{H}-\mathrm{SiC}$ wafers. The authors would also like to thank Dr. C. Jagadish of the Australian National University, Canberra, ACT 0200, Australia for assistance with Hall measurements.

${ }^{1}$ E. F. Schubert, in Semiconductors and Semimetals, edited by A. C. Gossard (Academic, New York, 1994), Vol. 40, p. 1.

${ }^{2}$ U. K. Misra, Y. F. Wu, B. P. Keller, S. Keller, and S. P. Denbaars, IEEE Trans. Microwave Theory Technol. 46, 756 (1998).

${ }^{3}$ T. Wang, Y. Ohno, M. Lachab, D. Nakagawa, T. Shirahama, S. Sakai, and H. Ohno, Appl. Phys. Lett. 74, 3531 (1999).

${ }^{4}$ J. H. Kim, G. M. Yang, S. C. Choi, J. Y. Choi, H. K. Cho, K. Y. Lim, and H. J. Lee, Jpn. J. Appl. Phys., Part 1 38, 681 (1999).

${ }^{5}$ Y. R. Yuan, K. Mohammed, M. A. Pudensi, and J. L. Merz, Appl. Phys. Lett. 45, 739 (1984)

${ }^{6}$ I. V. Kukushkin, K. von Klitzing, and K. Ploog, Phys. Rev. B 37, 8509 (1989).

${ }^{7}$ J. Wagner, A. Fischer, and K. Ploog, Phys. Rev. B 42, 7280 (1990).

${ }^{8}$ J. P. Bergman, T. Lundstorm, B. Monemar, H. Amano, and I. Akasaki, Appl. Phys. Lett. 69, 3456 (1996).

${ }^{9}$ H. K. Kwon, C. J. Eiting, D. J. H. Lambert, B. S. Shelton, M. M. Wong, T. G. Zhu, and R. D. Dupuis, Appl. Phys. Lett. 75, 2788 (1999).

${ }^{10}$ B. Shen, T. Someya, O. Moriwaki, and Y. Arakawa, Appl. Phys. Lett. 76, 679 (2000).

${ }^{11}$ S. Tanaka, S. Iwai, and Y. Aoyagi, Appl. Phys. Lett. 69, 4096 (1996).

${ }^{12}$ W. Götz, N. M. Johnson, C. Chen, H. Liu, C. Kuo, and W. Imler, Appl. Phys. Lett. 68, 3144 (1996).

${ }^{13}$ R. Dingle and M. Ilegems, Solid State Commun. 9, 175 (1971).

${ }^{14}$ W. G. Perry, M. B. Bremser, and R. F. Davis, J. Appl. Phys. 83, 469 (1998).

${ }^{15}$ The simulation program used, "1D POISSON" by G. L. Snider, is available through the Internet at http://www.nd/edu/ gsnider. Also see, I. H. Tan, G. L. Snider, L. D. Cheng, and E. L. Hu, J. Appl. Phys. 68, 4071 (1990). 